Modern Physics Letters A

(C) World Scientific Publishing Company

\title{
Spectroscopy and Thermodynamics of MSW Black Hole
}

\author{
Saneesh Sebastian* \\ Department of Physics, Cochin University of Science and Technology, Kochi 682022, India \\ saneeshphys@cusat.ac.in \\ V C Kuriakose \\ Department of Physics, Cochin University of Science and Technology, Kochi 682022, India \\ vck@cusat.ac.in
}

Received (Day Month Year) Revised (Day Month Year)

\begin{abstract}
We study the thermodvnamics and spectroscopy of a $2+1$ dimensional black hole proposed by Mandal et. a 1 . We put the background space time in Kruskal like co-ordinate and find period with respect to Euclidean time. Different thermodynamic quantities like entropy, specific heat, temperature etc are obtained. The adiabatic invariant for the black hole is found out and quantized using Bohr-Sommerfeld quantization rule. The study shows that the area spectrum of MSW black hole is equally spaced and the value of spacing is found to be $\hbar$

Keywords: Black hole, Thermodynamics, MSW black hole, entropy spectrum, area spectrum.

PACS Nos.: 04.70.Dy, 04.70.-s
\end{abstract}

\section{Introduction}

The black hole solution is one of the basic solutions of Einstein's General Theory of Relativity. The black hole solutions basically contain a singularity surrounded by an event horizon. Penrose proved mathematically the existence of singularity in the solutions of Einstein's General Theory of Relativity 2 . The horizon of black hole is mostly like a one sided membrane. It allows the passage of particles and radiation only in one direction. The black hole horizon has a thermodynamic behavior which was first showed by Bekenstein 3 . He showed that the area of the event horizon is a direct measure of black hole entropy. Later it was found that the four laws of black hole dynamics have a striking similarity with the four laws of thermodynamics. This similarity led Hawking to propose that black hole could emit particles 4 . Gravitational lensing is another important area of black hole physics $\frac{5617}{6}$

*Typeset names in 8 pt Times Roman, uppercase. Use the footnote to indicate the present or permanent address of the author. 
and a number of studies exist in literature on graviational lensing of black holes and naked singularities 89910 .

Bekenstein proposed that the black hole area is quantized and each quantum has a value $8 \pi l_{p}^{2}$ where $l_{p}$ is the Planck length and the spectrum is equally spaced. Bekenstein's idea is that there is a relation between the area spectrum and the quasinormal modes of Black holes 11 . Shahar Hod ${ }^{12}$ showed that the area spectrum is related to the imaginary part of quasinormal frequency and obtained a value $4 l_{p}^{2} \ln 3$. Later Maggiore showed that one should take both real and imaginary parts of the quasinormal modes when finding out the area quantum 13 . Maggiore obtained the same value for minimum area as Bekenstein obtained

There are number of works on the thermodynamics and spectroscopy of various types black holes. Periodicity and area spectrum of Schwarzschild and Kerr black hole are obtained by Zeng et. a14. Entropy and area spectrum of charged black hole using Quasinormal modes are studied by Wei et. a 15 . Kunstatter ${ }^{16}$ studied the area spectrum of higher diamensional black hole. Area spectrum of BTZ black hole is found using periodicity method by Larranaga 17

$2+1$ dimensional black holes acts as a toy model in Einstein gravity. Since the $2+$ 1 dimensional black hole are in reduced dimensions, the Einstein equation is exactly solvable. Both $2+1$ black hole and $3+1$ black hole share a number of properties and hence it is important to study simple $2+1$ black holes in Einstein gravity. Lower dimensional charged dilaton black hole has a relation to the lower dimensional string theory so it acts as a tool to study the lower dimensional string theory. The quasinormal modes of a charged dilaton black hole is studied by Fernando 18 and the area spectrum and thermodynamics are also studied 19 . Mandal et.al have obtained a one parameter family of black hole solution to classical dilaton system in two dimensions. This black hole is called MSW black hole in this work. Following Zeng 14 et. al we consider an out going wave which makes periodic motion outside the horizon. The gravitational system is periodic with respect to the Euclidean time with a period given by the inverse of Hawking temperature. The frequency of this out going wave is given by this temperature. Thus the adiabatic invariant term can be found out using surface gravity term. Using the method of Kunstatter 16 we have found the entropy and area spectrum of the MSW black hole.

In this paper we study the spectroscopy and thermodynamics of MSW black hole. The paper is organized as follows. In section. 2 we discuss the thermodynamic aspects of $2+1$ MSW black hole. In sction. 3 we discuss the area spectrum of the black hole. Finally, conclusion is given in section. 4

\section{Thermodynamics of MSW black hole}

The metric for MSW black hole is given by ( $c=G=1$ system of units)

$$
d s^{2}=-U(r) d t^{2}+\frac{d r^{2}}{U(r)}+\gamma^{2} r d \phi^{2}
$$


where $\mathrm{U}(\mathrm{r})$ is given by 20

$$
U(r)=8 \Lambda \beta r-2 M \sqrt{r} .
$$

This metric has a zero at (ie the horizon)

$$
r_{+}=\frac{M^{2}}{16 \Lambda^{2} \beta^{2}}
$$

where $\Lambda$ is the cosmological constant, $\beta$ is a constant factor, $M$ is the mass of the black hole. The black hole mass as a function of $r_{+}$is

$$
M=4 \Lambda \beta \sqrt{r_{+}} .
$$

The variation of $M$ versus $r_{+}$is shown in Fig.(1) for the different values of $\beta$ and

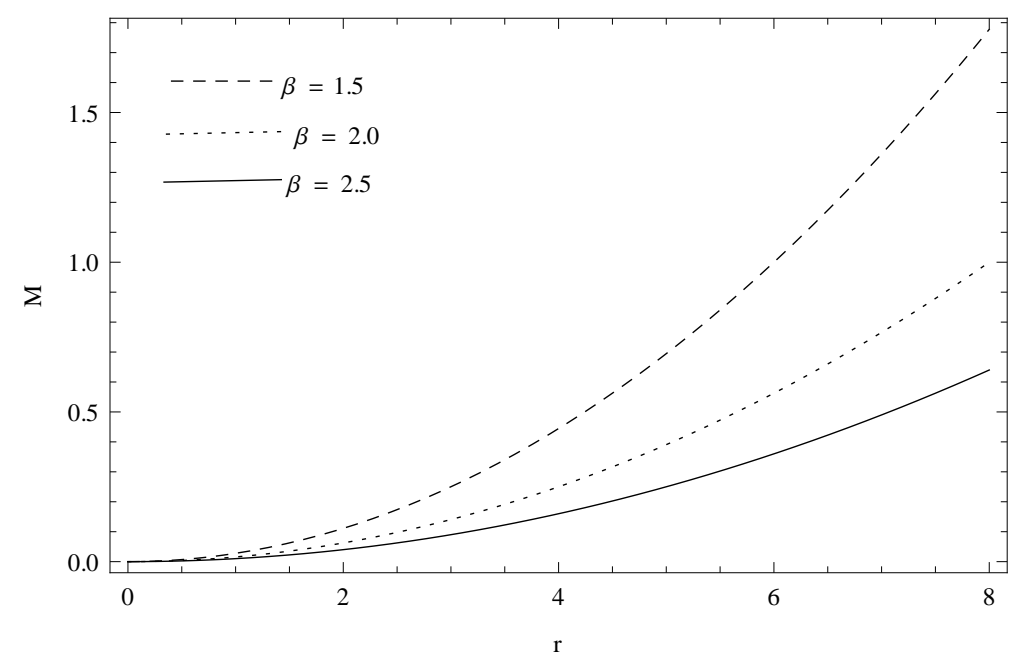

Fig. 1. Variation of mass with horizon radius for different values of $\beta$

it has a parabolic nature. The entropy $S$ of this $2+1$ dimensional black hole can be obtained as

$$
S=\frac{A}{4}=\frac{\pi r_{+}}{2} .
$$

Here $\mathrm{A}$ is the area, since it is a $2+1$ black hole area implies the circumference of the horizon. Since $r_{+}$can be expressed in terms of $M$, we can also express the entropy in terms of black hole mass, as

$$
S=\frac{\pi M^{2}}{32 \Lambda^{2} \beta^{2}} .
$$

Fig.(2) is a plot between entropy and mass and it shows a parabolic nature. The 


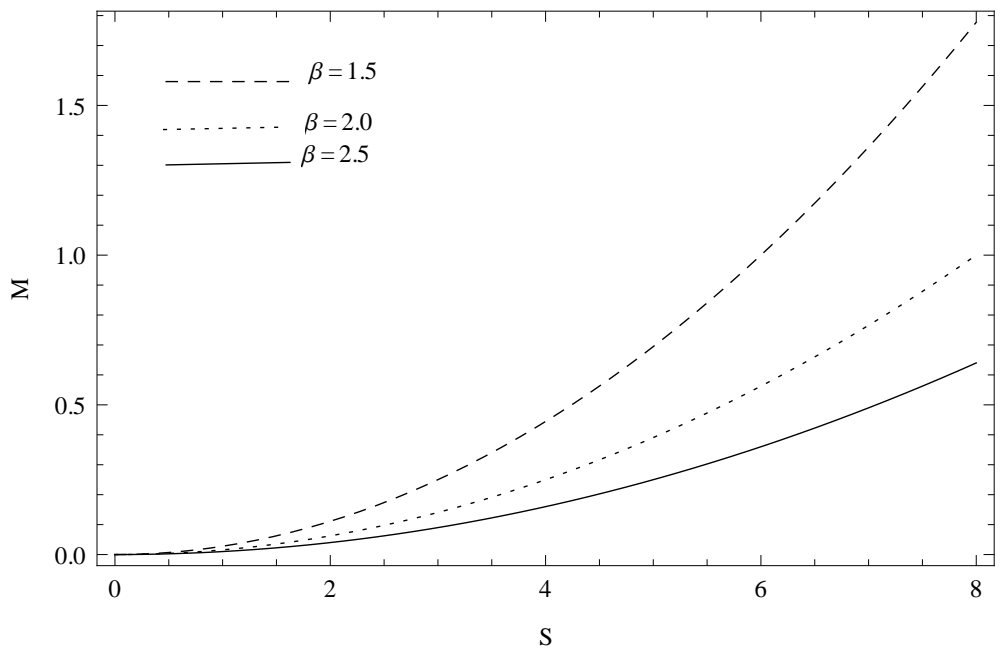

Fig. 2. variation of entropy with mass for diffrent values of $\beta$

black hole temperature can now be obtained since entropy-mass relation is known. The general expression for temperature $\mathrm{T}$ is

$$
T=\left(\frac{\partial M}{\partial S}\right)
$$

The heat capacity is given by the general expression

$$
C=T\left(\frac{\partial S}{\partial T}\right) .
$$

Eq.(5) can be inverted such that we get $r_{+}$in terms of $S$ as

$$
r_{+}=\frac{2 S}{\pi} \text {. }
$$

From Eq.(7) we can find out the temperature $\mathrm{T}$ as

$$
T=\frac{4 \Lambda \beta}{\pi \sqrt{r_{+}}}
$$

Fig.(3) shows variation of entropy with temperature. Entropy increases rapidly as temperature decreases. The specific heat of black hole can be obtained from Eq.(8). The specific heat is obtained as

$$
C=-\pi r_{+}=-2 S .
$$

The specific heat is negative as usual and is twice the entropy of the black hole. The negative heat capacity indicates that the black hole is thermodynamically unstable. For a large number of black holes heat capacity is negative showing that black holes are thermodynamically unstesble. 


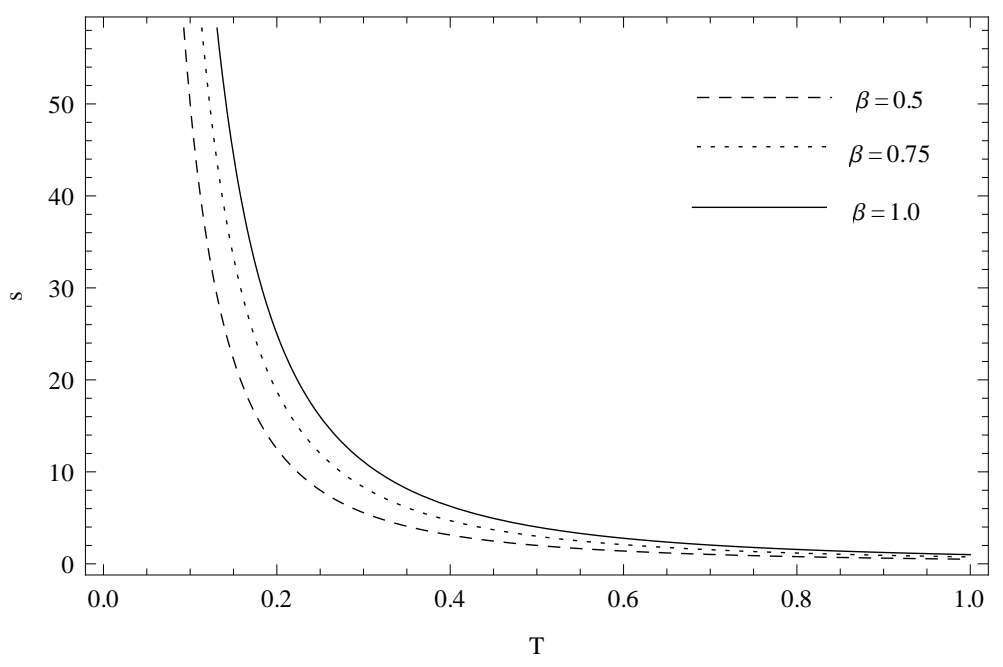

Fig. 3. variation of entropy with temperature for different values of $\beta$

\section{Spectroscopy of MSW black holes}

In this section we study the spectra of MSW black hole using adiabatic invariant method 2122 . We find the area spectrum by evaluating the adiabatic invariant integral which varies very slowly compared to the external perturbations. The adiabatic invariant is quantized using Bohr-Sommerfeld quantization rule. The first law of black hole thermodynamics says that

$$
d M=\frac{T_{H} d A}{4}
$$

We utilize the period of the motion of out going wave, which is shown to be related to the vibrational spectrum of the perturbed black hole, to quantize the area of the MSW black hole. It is well known that the gravity system is periodic with respect to the Euclidean time in Kruskal coordinate. Particles in this periodic gravitating system also own a period which is inverse of Hawking temperature. To find out area spectrum by periodicity method we use the Klein-Gordon equation given by

$$
g^{\mu \nu} \partial_{\mu} \partial_{\nu} \Phi-\frac{M^{2}}{\hbar^{2}} \Phi=0 .
$$

We define an action $A$ such that $\Phi$ is related to the action as

$$
\Phi=\exp \left[\frac{i}{\hbar} A(t, r)\right] .
$$

Adopting the wave equation ansatz for the scalar field we get the solution of wave equation. We can also obtain the solution from the Hamilton-Jacobi equation,

$$
g^{\mu \nu} \partial_{\mu} A \partial_{\nu} A+M^{2} A=0 .
$$


The action $A(t, r)$ can be decomposed into the functions of $r$ and $t$ and be written as $14 \mid 23$

$$
A(t, r)=-E t+W(r),
$$

where $E$ is the energy of the emitted particle measured by an observer at infinity. $W(r)$ in the action can be written in near horizon as 23

$$
W(r)=\frac{i \pi E}{f^{\prime}\left(r_{+}\right)},
$$

where we consider the out going wave near the horizon. In this case, it is obivious that the wave function $\Phi$ outside the horizon can be expressed in the form

$$
\Phi=\exp \left[-\frac{i E t}{\hbar}\right] \Psi\left(r_{+}\right)
$$

and $\Psi\left(r_{+}\right)$is given by

$$
\Psi\left(r_{+}\right)=\exp \left[\frac{\pi E}{h f^{\prime}\left(r_{+}\right)}\right],
$$

From Eq.(18) we can find that $\Phi$ is periodic with a period

$$
\tau=\frac{2 \pi \hbar}{E} \text {. }
$$

The gravitating system is periodic in Kruskal coordinate in Euclidean time and the moving particle also gets a periodic motion in this periodic gravity. The period is related inversely to the Hawking temperature as

$$
\tau=\frac{2 \pi}{\kappa_{r}}=\frac{\hbar}{T_{H}}
$$

and hence, the Hawking temperature is given by

$$
T_{H}=\frac{\hbar \kappa_{r}}{2 \pi} .
$$

In the near horizon

$$
U(r)=-M+4 \Lambda \beta \sqrt{r_{+}} .
$$

As $U(r) \rightarrow 0$, the change in the mass $d M$, becomes

$$
d M=2 \Lambda \beta \frac{d r_{+}}{\sqrt{r_{+}}} .
$$

The adiabatic invariant ${ }^{22}$ is defined as

$$
I=\int \frac{2 \pi d M}{\kappa_{r_{+}}}
$$

where $\kappa_{r_{+}}$is the surface gravity and is given by

$$
\kappa_{r_{+}}=\left.\frac{d U(r)}{d r}\right|_{r=r_{+}} .
$$


From (23)

$$
\left.\frac{d U(r)}{d r}\right|_{r=r_{+}}=\frac{4 \Lambda \beta}{2 \sqrt{r_{+}}}
$$

and using Eq.(26), we can have

$$
\kappa_{r_{+}}=\frac{2 \Lambda \beta}{\sqrt{r_{+}}}
$$

Substituting Eqs.(28) and (24) in Eq.(25) and applying Bohr-Sommerfeld quantization rule, we get

$$
2 \pi r_{+}=A_{n}=n \hbar
$$

and $\Delta A$ is given by

$$
\Delta A=A_{n+1}-A_{n}=\hbar
$$

Thus we see that the circumference spectrum of MSW black hole is discrete and the spacing is equidistant. For this system the circumference spectrum is indepedent of black hole parameters. Since the entropy of this system is related to circumference spectrum, it is also quantized.

\section{Conclusion}

The thermodynamics of $2+1$ dimensional MSW black holes are studied and area spectrum is obtained using adiabatic invariant method. We have obtained the heat capacity, temperature and mass of MSW black hole. (The different quantities are plotted and studied their behavior) The mass of the black hole is plotted for various values of horizon radius with different parameter $\beta$ and the graph shows that the horizon radius increases quadratically with mass, the same behavior is shown for mass versus entropy. The variation of temperature with entropy is also plotted and the graph shows that the entropy increases very rapidly with decreasing temperature. The heat capacity is found to be negative as is the case for most black holes. This black hole does not show any phase transition. The period of Euclidean time is found out and adiabatic invariant is calculated. Using Bohr-Sommerfeld quantization rule we have obtained the area spectrum, here the circumference spectrum and is found to be quantized and equally spaced.

\section{Acknowledgement}

SS wishes to thank CSIR, New Delhi for financial support under CSIR-SRF scheme. VCK wishes to acknowledge Associateship of IUCAA, Pune, India

\section{References}

1. G. Mandal et. al Mod. Phys. Lett. A 61685 (1991)

2. R.Penrose, Structure of space time, W. A. Benjamin N. Y (1968) 
3. J. D. Bekenstein,Phys. Rev. D 7, 2333 (1973)

4. S. W. Hawking Commun. Math. Phys. 43 199-220 (1975)

5. K. S. Virbhadra et. al Phys. Rev. D 62084003 (2000)

6. K. S. Virbhadra et. al Phys. Rev. D 65103004 (2002)

7. K. S. Virbhadra et. al Phys. Rev. D 77124014 (2008)

8. K. S. Virbhadra et. al Astron. Astrophys 337 (1998)1-8

9. K. S. Virbhadra et. al. Phys. Rev. D 79083004 (2009)

10. C. M. Claudel et. al J. Math. Phys. 42 818-838 (2001)

11. J. D. Bekenstein, Lett . Nuovo Cimento Soc. Ital. fis. 11467 (1974)

12. S. Hod Class. Quantum grav. 23 (2006)

13. M. Maggiore Phys.Rev. Lett. 100 141301(2008)

14. X. Zeng et. al Eur.Phys. J. C 72 (2012)

15. S. W. Wei et. al Phys. Rev. D 81104042 (2010)

16. G. Kunstatter, Phys. Rev. Lett. 90162301 (2003)

17. A. Larranaga Int. J. Mod. Phys. D 211250068 (2012)

18. S. Fernando, Gen. Rel. Gravitation 36 71-82 (2004)

19. R. Tharanath and V. C. Kuriakose DOI 10.1007/s10714-013-1554-5

20. K. C. K. Chan, R. B. Mann Phys. Rev. D. 506385 (1994)

21. Qing-Quan Jiang and Yan Han, Phys. Lett. B 718 (2012) 584-588

22. Qing-Quan Jiang, arXiv:gr-qc 1210.4397v1

23. D. Y. Chen et.al Phys. Lett. B 665 (2008) 106 